\title{
Implementación de una planta de elaboración de bebida de papaya (Carica papaya) con linaza (Linum usitatissimum)
}

\author{
Karen Nathaly Urquizo Baldarrago* \\ José Alonso Pardo Delgado** \\ Universidad de Lima. Lima, Perú
}

Recibido: 23 de abril de 2015 / Aprobado: 13 de julio de 2015

\begin{abstract}
RESUMEN: La papaya es una fruta tropical con muchas propiedades medicinales y un gran sabor, que se utiliza para elaborar postres, jugos y helados. La linaza también presenta posee nutricionales, como el uso de la semilla para tratar problemas digestivos. Según el informe de Geosalud (Van Saafelo, 2012), en el Perú el 34 \% de personas sufre de hipertensión arterial, porque tienen un alto grado de colesterol y de triglicéridos. Los nutricionistas recomiendan la papaya y la linaza porque permiten reducir dichos niveles; además, facilitan la digestión, evitan la gastritis y mejoran la calidad de la piel.
\end{abstract}

Palabras clave: papaya / linaza / jugos de fruta / digestión

\section{/ hipertensión arterial}

Implementation of a Elaboration Plant of a Papaya (Carica papaya) Beverage with Linseed (Linum usitatissimum)

ABstract: The papaya is a tropical fruit with many medicinal properties and a great flavor, used to prepare desserts, juices and ice creams. In addition it is worth mentioning that the flaxseed also presents different nutritional properties within which highlights the use of the seed for digestive problems. According to the report from Geosalud (Van Saafelo, 2012), 34\% of people suffer from high blood pressure in Peru. This is because individuals have high levels of cholesterol and triglycerides. Nutritionists recommend that within the food that should be consumed to reduce these levels are papaya and flaxseed; moreover, these two foods facilitate digestion, prevent gastritis and improve skin quality.

Key words: papaya / flaxseed / fruit juices / digestion / hypertension

Correos electrónicos: *Kurquizo@ulima.edu.pe, **josea_123@hotmail.com 


\section{INTRODUCCIÓN}

Actualmente es cada vez mayor el interés de la población por los temas referidos a la salud; especialmente por la alimentación sana y natural. Así, es notorio el aumento del número de personas que se preocupan por consumir alimentos elaborados a partir de las frutas, que les ayude a tener una vida más saludable (Maximixe, 2012). Además de las frutas también se ha incrementado el consumo de semillas como la linaza. Ello se puede observar por el mayor ingreso en el mercado de yogurt, cereales, galletas integrales, entre otros productos conocidos por sus propiedades saludables.

Por otro lado, las estadísticas de producción de jugos, néctares y bebidas de fruta muestran un constante crecimiento desde el año 2002 hasta el 2012, con excepción del año 2009, cuando la crisis económica provocó una reducción del crecimiento del PBI a 0,9 \% (BCRP, 2012). Este crecimiento sostenido pone en evidencia un mayor consumo de los productos que tienen como materia prima las diversas frutas existentes en el mercado peruano (Ministerio de la Producción, 2012).

Por ello, la presente investigación está referida a la fabricación de una bebida obtenida de la mezcla de papaya con linaza, condición que la diferencia frente a los néctares de frutas, ya que contiene vitaminas $\mathrm{A}, \mathrm{B}$ y $\mathrm{C}$, así como potasio, magnesio, calcio, hierro y omega 3 (Aravind, Debjit Bhowmik, Duraivel y Harish, 2013).

\section{ESTUDIO DE MERCADO}

\subsection{Usos y características del producto}

El producto está orientado a las personas interesadas en ingerir bebidas preparadas con frutas que presenten un contenido de vitaminas, proteínas y fibra, que pueden ser usadas con fines médicos o regímenes de dieta para la salud (Aravind et al., 2013), para hidratarse o como acompañamiento en las comidas.

En cuanto a las propiedades de la bebida de papaya con linaza, esta presenta un aspecto claro, límpido y brillante; su color es ligeramente anaranjado, tiene el olor característico de la papaya; provoca una sensación de frescura; es ligeramente dulce, con predominio del sabor de la fruta, y con una consistencia un poco viscosa; contiene vitami- 
nas A, C, complejo B, potasio, hierro, magnesio, fibra, calcio, omega 3 y fibra dietética.

\subsection{Determinación del área geográfica del estudio}

El estudio se enfoca específicamente en los segmentos A, B y C del mercado peruano, por ser los segmentos que presentan un mayor gasto en alimentación (Ipsos Apoyo, 2012c). Asimismo, se considera todo el territorio nacional, porque el producto puede ser consumido en diversos departamentos del país.

\subsection{Análisis de la demanda}

El producto expuesto no cuenta con una demanda histórica, porque es un producto innovador en el mercado peruano, que hasta el momento no ha sido fabricado ni comercializado en el país. Sin embargo, para el presente trabajo se toma en cuenta la información referente a la demanda de los jugos, néctares y refrescos envasados listos para consumir, pues estos son sustitutos del que se proyecta elaborar; por lo que para el cálculo de la demanda interna aparente (DIA) se utiliza información de la producción y de las importaciones y exportaciones de tales productos.

Tabla 1

Demanda interna aparente - Perú

\begin{tabular}{rrrrr}
\hline Año & $\begin{array}{c}\text { Producción } \\
\text { (litros) }\end{array}$ & $\begin{array}{c}\text { Importaciones } \\
\text { (litros) }\end{array}$ & $\begin{array}{c}\text { Exportaciones } \\
\text { (litros) }\end{array}$ & $\begin{array}{c}\text { Demanda interna } \\
\text { aparente (litros) }\end{array}$ \\
\hline 2005 & 63264052,45 & 230000,00 & 4700000,00 & 58794052,45 \\
2006 & 107186009,99 & 440000,00 & 7500000,00 & 100126009,99 \\
2007 & 216963073,13 & 770000,00 & 9800000,00 & 207933073,13 \\
2008 & 292532856,94 & 648400,00 & 11011100,00 & 282170156,94 \\
2009 & 287011147,45 & 1163100,00 & 15119500,00 & 273054747,45 \\
2010 & 310036120,58 & 1322900,00 & 2964400,00 & 290394620,58 \\
2011 & 337616102,90 & 1316600,00 & 24599800,00 & 314332902,90 \\
2012 & 363483234,37 & 1753711,20 & 10012118,60 & 355224826,97 \\
\hline
\end{tabular}

Fuente: Maximixe (2012), Ministerio de la Producción (2012)

Elaboración propia 
A continuación se procedió a proyectar la DIA de jugos, néctares y refrescos para los años 2012-2023, como se observa en la tabla 3. Para el cálculo se tomó como variante independiente el Producto Bruto Interno del Perú, y se realizó un análisis de diversas regresiones, como se observa en la tabla 2.

Tabla 2

Regresiones y coeficiente de determinación (PBI vs. demanda)

\begin{tabular}{lcc}
\hline \multicolumn{1}{c}{ Tendencia } & $\mathbf{R}^{\mathbf{2}}$ & Función \\
\hline Exponencial & 0.7741 & $6361206 * \mathrm{e}^{1.8000^{* 10^{-8}} * \mathrm{X}}$ \\
Lineal & 0.8888 & $-376318128,849+301734 * \mathrm{X}$ \\
Logarítmica & 0.9232 & $-11502656,750+61567229,548^{*} \mathrm{LN}(\mathrm{X})$ \\
Potencial & 0.8266 & $9.5729 * 10^{-22} * \mathrm{X}^{3.5423}$ \\
\hline
\end{tabular}

Elaboración propia

Dentro de estas se optó por la regresión logarítmica, debido a que presenta el coeficiente de determinación más alto, tal como se observa en la figura 1.

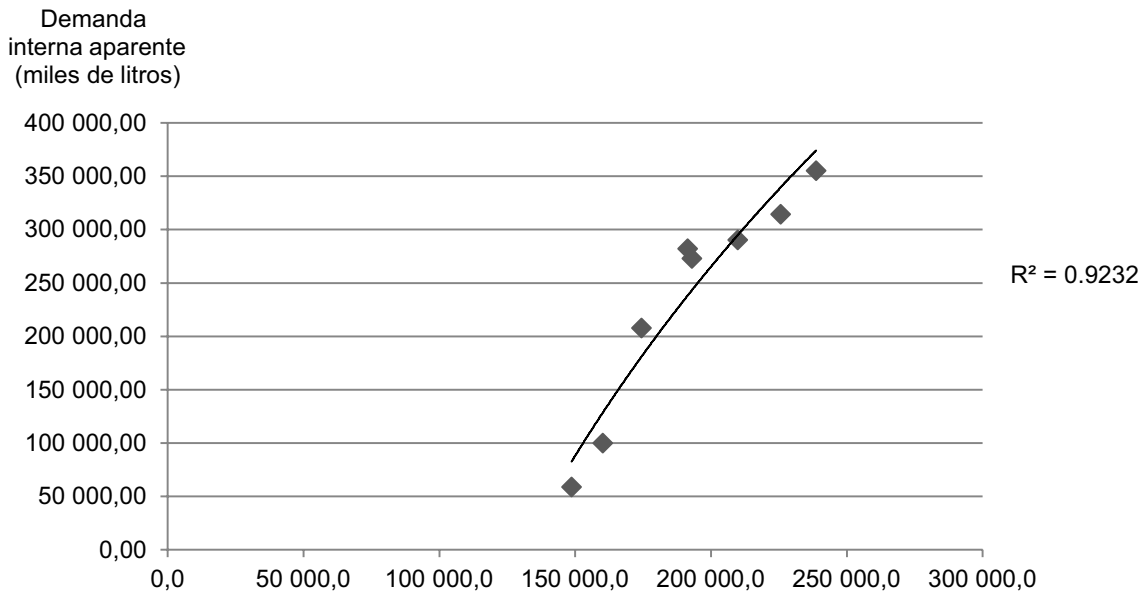

Figura 1. Demanda interna aparente y línea de tendencia Elaboración propia 
Tabla 3

Proyección de la demanda - Perú

\begin{tabular}{ccc}
\hline Año & $\begin{array}{c}\text { Producto bruto interno } \\
\text { (soles) }\end{array}$ & $\begin{array}{c}\text { Demanda interna aparente } \\
\text { (litros) }\end{array}$ \\
\hline 2014 & 266702994,52 & 442495041,13 \\
2015 & 281905065,21 & 476624927,89 \\
2016 & 297973653,92 & 510754814,65 \\
2017 & 312872336,62 & 540793807,76 \\
2018 & 328515953,45 & 570832800,86 \\
2019 & 344941751,12 & 600871793,97 \\
2020 & 362188838,68 & 630910787,07 \\
2021 & 380298280,61 & 660949780,18 \\
2022 & 399313194,64 & 690988773,28 \\
2023 & 419278854,38 & 721027766,39 \\
\hline
\end{tabular}

Fuente: INEI (s. f.a)

Elaboración propia

\subsection{Demanda del proyecto}

Se realizó el análisis de las cuatro variables más importantes, como se muestran a continuación:

a) Segmentación geográfica

El producto será comercializado y distribuido en Lima metropolitana, puesto que, de acuerdo con el IGM Poblacional 2012 de Ipsos Apoyo (2012a), alrededor del 31,44 \% de la población peruana se concentra en esta área geográfica del país. Asimismo, Lima metropolitana es la región donde se concentra el mayor porcentaje de población dentro de los niveles socioeconómicos A, B y C, según Ipsos Apoyo (2012d).

b) Segmentación psicográfica

El producto estará enfocado, de acuerdo al informe de niveles socioeconómicos (Ipsos Apoyo (2012d), a los "niveles socioeconómicos $\mathrm{A}, \mathrm{B}$ y $\mathrm{C}$, debido a que esta población se ve más atraída hacia el consumo de productos saludables" (Ipsos Apoyo, 2012c). Además, se eligió estos niveles por ser los de mayor poder adquisitivo; y como se muestra en la tabla 4, son los hogares de este sector los que gastan más dinero en la alimentación dentro del hogar. 
Tabla 4

Gasto mensual en el hogar en soles por niveles socioeconómicos

\begin{tabular}{lcccccc}
\hline & & \multicolumn{5}{c}{ Nivel socioeconómico } \\
\cline { 3 - 7 } Respuestas & Total S/. & A & B & C & D & E \\
& & S/. & S/. & S/. & S/. & S/. \\
\hline Alimentación & 919 & 2037 & 1175 & 858 & 730 & 693 \\
Dentro del hogar & 657 & 1358 & 850 & 629 & 535 & 511 \\
Fuera del hogar & 262 & 679 & 325 & 229 & 195 & 182 \\
\hline
\end{tabular}

Fuente: Ipsos Apoyo (2012d)

Elaboración propia

c) Segmentación demográfica

La bebida de papaya con linaza está orientada a consumidores que tengan más de 13 años de edad, debido a que, según la encuesta realizada, el producto tiene atracción para los encuestados dentro de ese rango de edades. Además, según IGM tendencias en salud y alimentación (Ipsos Apoyo, 2012c), las personas que tienen este mismo rango de edad son las que se preocupan más por consumir alimentos sanos y saludables.

d) Segmentación conductual

Se determinó como el consumidor objetivo aquel que se encuentra interesado en comprar jugos envasados dentro de los niveles socioeconómicos explicados anteriormente; para lo cual se tomó en cuenta la penetración de mercado de jugos envasados, extraído de Ipsos Apoyo "Liderazgo de productos comestibles 2012". A continuación, en la tabla 5 se presenta la penetración de jugos envasados por nivel socioeconómico.

Los porcentajes tomados en cada una de las variables se detallan en la tabla 6.

Además, para elegir el mercado meta se ha considerado la intención de compra y la intensidad de compra, cuyos valores, según la encuesta, son $81,82 \%$ y $62,97 \%$, respectivamente.

Asimismo, se ha tomado en cuenta que el proyecto va a capturar el $2 \%$ de participación del mercado segmentado previamente. Este porcentaje de participación se ha calculado tomando en cuenta que la em- 
presa va a capturar el promedio ponderado de las participaciones que tienen las otras marcas en los niveles socioeconómicos A, B y C.

Tabla 5

Penetración de productos

\begin{tabular}{lccccccc}
\hline \multirow{2}{*}{ Productos } & $\mathbf{2 0 1 1}$ & $\mathbf{2 0 1 2}$ & \multicolumn{6}{c}{ Nivel socioeconómico (\%) } \\
& (\%) & $\mathbf{( \% )}$ & A & B & C & D & E \\
\hline Jugos envasados & 48 & 51 & 52 & 58 & 46 & 48 & 63 \\
\hline
\end{tabular}

Fuente: Ipsos Apoyo (2012e)

Elaboración propia

Tabla 6

Porcentajes tomados en cada criterio de segmentación

\begin{tabular}{ccccccccc}
\hline Año & $\begin{array}{c}\text { Pobl. } \\
\text { Lima } \\
\%\end{array}$ & $\begin{array}{c}\text { NSE A } \\
\%\end{array}$ & $\begin{array}{c}\text { Penetr. } \\
\text { NSE A } \\
\%\end{array}$ & $\begin{array}{c}\text { NSE B } \\
\%\end{array}$ & $\begin{array}{c}\text { Penetr. } \\
\text { NSE B } \\
\%\end{array}$ & $\begin{array}{c}\text { NSE C } \\
\%\end{array}$ & $\begin{array}{c}\text { Penetr. } \\
\text { NSE C } \\
\%\end{array}$ & $\begin{array}{c}\text { Población } \\
\mathbf{1 3 - 6 5 +} \\
\%\end{array}$ \\
\hline 2014 & 31,44 & 5,1 & 52 & 16,10 & 58 & 34,90 & 46 & 78,10 \\
2015 & 31,58 & 5,1 & 52 & 16,10 & 58 & 34,90 & 46 & 78,10 \\
2016 & 31,71 & 5,1 & 52 & 16,10 & 58 & 34,90 & 46 & 78,10 \\
2017 & 31,85 & 5,1 & 52 & 16,10 & 58 & 34,90 & 46 & 78,10 \\
2018 & 31,98 & 5,1 & 52 & 16,10 & 58 & 34,90 & 46 & 78,10 \\
2019 & 32,12 & 5,1 & 52 & 16,10 & 58 & 34,90 & 46 & 78,10 \\
2020 & 32,25 & 5,1 & 52 & 16,10 & 58 & 34,90 & 46 & 78,10 \\
2021 & 32,38 & 5,1 & 52 & 16,10 & 58 & 34,90 & 46 & 78,10 \\
2022 & 32,51 & 5,1 & 52 & 16,10 & 58 & 34,90 & 46 & 78,10 \\
2023 & 32,63 & 5,1 & 52 & 16,10 & 58 & 34,90 & 46 & 78,10 \\
\hline
\end{tabular}

Fuente: INEI (s. f.a), Ipsos Apoyo (2012a)

Elaboración propia

En la tabla 7 se puede observar los datos de participación de cada empresa de acuerdo a los niveles socioeconómicos. 
Tabla 7

Marca de jugos envasado que consume habitualmente

\begin{tabular}{lccccc}
\hline \multirow{2}{*}{$\begin{array}{l}\text { Marca consumida } \\
\text { habitualmente }\end{array}$} & $\begin{array}{c}\text { A } \\
\text { \% }\end{array}$ & $\begin{array}{c}\text { B } \\
\%\end{array}$ & $\begin{array}{c}\text { C } \\
\%\end{array}$ & $\begin{array}{c}\text { D } \\
\%\end{array}$ & $\begin{array}{c}\text { E } \\
\%\end{array}$ \\
\hline Tampico & 9 & 2 & 2 & 3 & 6 \\
Laive & 11 & 1 & 3 & 2 & 0 \\
Otras marcas & 2 & 6 & 3 & 1 & 0 \\
\hline
\end{tabular}

Elaboración propia

Finalmente, para determinar la demanda del proyecto se ajusta la demanda interna aparente proyectada mostrada en la tabla 3 , con los porcentajes determinados en el análisis anterior. En la tabla 8 se presenta el resultado de la demanda del proyecto para cada año.

Tabla 8

Demanda del proyecto (litros y botellas)

\begin{tabular}{rccccc}
\hline & $\begin{array}{c}\text { Demanda interna } \\
\text { aparente de jugos, }\end{array}$ & $\begin{array}{c}\text { Demanda interna } \\
\text { aparente de jugos } \\
\text { envasados }\end{array}$ & & \multicolumn{2}{c}{ Demanda del proyecto } \\
\cline { 5 - 6 } & $\begin{array}{c}\text { néctares y refrescos } \\
\text { (litros) }\end{array}$ & $\begin{array}{c}\text { Lima metropolitana } \\
\text { NSE A, B, C (litros)* }\end{array}$ & Litros & & Botellas \\
\hline 2014 & 442495041,13 & 39019102,06 & 314015,78 & 1060864,11 \\
2015 & 476624927,89 & 42213722,63 & 339725,27 & 1147720,50 \\
2016 & 510754814,65 & 45426793,45 & 365583,24 & 1235078,52 \\
2017 & 540793807,76 & 48302981,19 & 388730,07 & 1313277,25 \\
2018 & 570832800,86 & 51201102,75 & 412053,41 & 1392072,33 \\
2019 & 600871793,97 & 54119947,89 & 435543,53 & 1471430,85 \\
2020 & 630910787,07 & 57058071,46 & 459188,80 & 1551313,51 \\
2021 & 660949780,18 & 60015234,52 & 482987,29 & 1631713,83 \\
2022 & 690988773,28 & 62991854,16 & 506942,37 & 1712643,14 \\
2023 & 721027766,39 & 65987270,88 & 531048,72 & 1794083,51 \\
\hline
\end{tabular}

*DIA * $\%$ Pob.Lima *\% NSE A *\% Pen.NSE A+ \%Pob.Lima *\% NSE B *\% Pen.NSE B + \% Pob.Lima *\% NSE $C * \%$ Pen.NSE C)

**DIA(NSE A,B,C) *\% Pob.13-+65 *\% Intención.compra *\% Intensidad.compra

*\% Participación

Elaboración propia 


\section{LOCALIZACIÓN DE PLANTA}

Para determinar la óptima localización geográfica de la planta se tomaron en cuenta factores determinantes, como la disponibilidad de materias primas, la cercanía al mercado, la disponibilidad de mano de obra, el abastecimiento de energía eléctrica, el abastecimiento de agua potable, los servicios de transporte y la disponibilidad de terrenos. De acuerdo con estos parámetros, se establecieron como posibles ubicaciones las regiones de Lima, La Libertad y Ucayali.

Con la finalidad de establecer la mejor macrolocalización entre estas regiones se aplicó el método de ranking de factores, teniendo en cuenta los mencionados en el párrafo anterior. Producto de la evaluación resultó que la mejor opción para ubicar la planta es la región de Lima.

A continuación se realizó el análisis de microlocalización, en el que se consideraron los distritos de Ate, Lurín e Independencia, debido a que son los distritos que presentan una mayor oferta de propiedades industriales, por lo que se aplicó nuevamente el método de ranking de factores, de donde se obtuvo que la opción más adecuada para ubicar la planta es el distrito de Ate; principalmente por la proximidad a la materia prima, a la disponibilidad de mano de obra y al abastecimiento de energía eléctrica.

\section{TAMAÑO DE PLANTA}

Para poder determinar el tamaño de planta se analizaron las relaciones tamaño-mercado, tamaño-recursos productivos, tamaño-tecnología y tamaño-punto de equilibrio; cuyo resultado se observa en la tabla 9:

Tabla 9

Selección del tamaño de planta

\begin{tabular}{lc}
\hline \multicolumn{1}{c}{ Factor } & $\begin{array}{c}\text { Tamaño en litros } \\
\text { por hora }\end{array}$ \\
\hline Tamaño-mercado & 74 \\
Tamaño-recursos productivos & 170,45 \\
Tamaño-tecnología & 125,42 \\
Tamaño-punto de equilibrio & 20,46 \\
\hline
\end{tabular}

Elaboración propia 
Al comparar los resultados, se concluye que la limitante respecto de la demanda del mercado es el recurso tamaño-tecnología, por lo que se concluye que el tamaño y la capacidad de planta sería de $125,42 \mathrm{l} / \mathrm{h}$.

\section{INGENIERÍA DEL PROYECTO}

\subsection{Definición del producto basada en sus características de fabricación}

Linpawer es una bebida que se obtiene a partir de la combinación de pulpa de papaya con gel de linaza, azúcar, ácido cítrico y agua, según las proporciones detalladas en la tabla 10, y luego de pasar por operaciones y procesos térmicos se obtiene el producto final, envasado en botella de vidrio de $296 \mathrm{ml}$, que cumple con los controles de calidad, como se observa en la figura 2 .

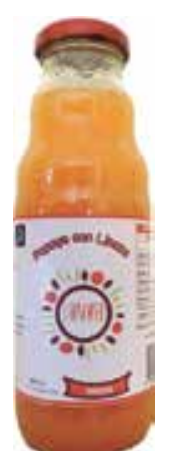

Figura 2. Bebida de papaya con linaza

Elaboración propia
Tabla 10

Composición de bebida de papaya con linaza

\begin{tabular}{lc}
\hline \multicolumn{1}{c}{ Insumo } & Composición \% \\
\hline Pulpa de papaya & 48,01 \\
Gel de linaza & 16,80 \\
Agua & 30,01 \\
Azúcar & 5,10 \\
Ácido cítrico & 0,07 \\
\hline
\end{tabular}

Elaboración propia

\subsection{Descripción del proceso de producción}

El proceso productivo consta de 15 etapas, que se detallan a continuación:

a) Inspección y selección de la materia prima

Se inicia con la llegada de los camiones que traen la papaya y la linaza. Una vez recibida la materia prima requerida para la producción diaria, esta pasa a la primera tarea, que consiste en eliminar las papayas defectuosas, para lo cual se hace un control manual y visual para desechar aquellas que presenten magulladuras, hongos, 
golpes y otros defectos que puedan afectar la calidad del producto final; en el caso de la linaza, la selección se realiza por medio de una inspección visual para separar las semillas de la linaza de cualquier impureza que se encuentre en esta. En esta tarea, la pérdida es de alrededor del $2 \%$ de las papayas y $0,5 \%$ de la linaza.

b) Pesado de la materia prima

Una vez seleccionada la materia prima, un operario se encarga de pesar las papayas y la linaza con el fin de determinar la cantidad que ingresará al proceso productivo. Por lo tanto, permitirá establecer los rendimientos y controlar los indicadores de producción.

c) Lavado

La papaya y la linaza son transportadas por medio de un carro estantería a la estación de lavado, donde de manera manual se lavan las papayas en una tina, con solución de hipoclorito de sodio (3 $\mathrm{ml}$ de solución de hipoclorito de sodio al $3 \%$ por cada 100 litros de agua). Esta operación se realiza con la finalidad de eliminar los restos de tierra que puedan estar adheridos en la fruta.

En el caso de la linaza, esta se coloca en un colador industrial para pasarla por un lavado de chorro de agua con presión constante. En esta operación se pierde el $1 \%$ del peso, tanto de la papaya como de la linaza.

d) Pelado, limpiado y cortado

Una vez lavada la papaya, se traslada por medio de un carro estantería a la zona de pelado. Debido a la forma y delicadeza de la papaya, esta se pela y limpia manualmente, quitándole las cáscaras y pepas. En esta operación se pierde aproximadamente el $22,73 \%$ de papaya.

e) Escaldado

La fruta lavada es transportada al proceso de escaldado por medio del carro estantería. Luego, un operario introduce la papaya en un equipo de escaldado. En este proceso se controla que la papaya se someta a una temperatura de 70 a $75^{\circ} \mathrm{C}$ durante dos minutos, con el fin de eliminar la mayor cantidad de carga bacteriana.

\section{f) Pulpeado}

Una vez concluido el proceso de escaldado la papaya es transportada en un carro estantería a la estación de pulpeado, donde a través de la máquina despulpadora se obtiene la pulpa de papaya, libre de cáscaras y pepas. En el nivel industrial la operación se realiza en 
despulpadoras industriales. Luego, la pulpa es trasladada por medio de tuberías de acero inoxidable a un tanque de almacenamiento temporal, en la zona de mezclado.

g) Cocido en marmita

Después de lavada la linaza se coloca en la mesa de acero inoxidable con inclinación, para luego ser introducida en la marmita con una paleta. Al mismo tiempo, se dosifica una cantidad de agua para hervir la linaza por aproximadamente diez minutos, de esta manera se obtiene el gel de linaza, que se utilizará en los siguientes procesos.

h) Filtrado

El gel de linaza, junto con las semillas, se traslada por un túnel hasta el cernidor vibratorio de granulometría apropiada, que consiste en separar el gel de las semillas, las que se depositarán en dos tanques. El gel de linaza se traslada por medio de tuberías de acero inoxidable al tanque de almacenamiento temporal en la zona de mezclado; mientras que las semillas de linaza se trasladan a la operación de cocción, donde se realiza un reproceso. En esta estación se pierde alrededor del 39,07 \% de peso.

i) Mezclado

Una vez que la pulpa de papaya se encuentra en el tanque de almacenamiento, esta se dosifica al tanque de mezclado con paletas. Luego se dosifica el gel de linaza, azúcar, ácido cítrico y el agua tratada, la cual pasa por los procesos de esterilizado ultravioleta y filtrado, en los cuales se reduce su carga bacteriana, según la composición establecida en la tabla 10. Se ha encontrado que en este proceso se pierde un $1,5 \%$ del peso.

j) Pasteurizado

Una vez obtenida la mezcla, esta solución pasa por medio de tuberías de acero inoxidable al proceso de pasteurizado. Esta operación se realiza con la finalidad de reducir la carga microbiana, asegurar la inocuidad del producto y alargar su vida útil. En este proceso el operario tiene que controlar que la temperatura sea entre $\approx 75$ y $85^{\circ} \mathrm{C}$, y luego disminuirá hasta $\approx 40$ y $45^{\circ} \mathrm{C}$.

k) Envasado

Una vez que la mezcla ha sido pasteurizada, esta pasa al proceso de envasado por medio de tuberías de acero inoxidable. Por otro lado, los envases ya inspeccionados y lavados llegan a la mesa de envasado de botellas por medio de una faja transportadora, luego el 
operario toma tres botellas y realiza con la máquina envasadora la dosificación en caliente de $296 \mathrm{ml}$ de bebida por envase. Para esta operación se requiere de un trabajador que se encarga de manejar la máquina envasadora, que es semiautomática.

\section{1) Tapado}

Tras ser envasado el producto, los envases son colocados en una faja transportadora, para luego ser tapadas al vacío con la tapadora semiautomática con ayuda de un operario, con el objetivo de que la bebida mantenga sus propiedades.

m) Enfriado

Una vez realizado el tapado al vacío, las botellas son colocadas en la estación de enfriado, pasando por un túnel de enfriamiento, con el objetivo de disminuir la temperatura del producto.

n) Etiquetado

Después de la operación de enfriado, las botellas envasadas son colocadas en una mesa de acopio para luego pasar al proceso de etiquetado, en el que por medio de una máquina el operario coloca las etiquetas impresas. Cabe resaltar que las etiquetas deben contener el nombre del alimento, la declaración de los ingredientes y aditivos empleados en la elaboración del producto, el nombre y la dirección de fabricante, el número de registro sanitario, las condiciones especiales de conservación y el contenido neto, que son los requerimientos que señala la guía para el etiquetado de alimentos envasados (Indecopi, 2008).

o) Codificado

Las botellas etiquetadas pasan, mediante una faja transportadora, al proceso de codificado, donde con la codificadora de tinta se le coloca la fecha de vencimiento y el número de lote.

p) Embalado y sellado

La bebida de papaya con linaza pasa por medio de una faja transportadora al área de embalaje, donde un operario coloca manualmente 24 botellas por caja y las sella. Luego las cajas son transportadas al almacén de producto terminado.

En la figura 3 se observa el diagrama de operaciones completo para la elaboración de la bebida de papaya con linaza, de $296 \mathrm{ml}$. 


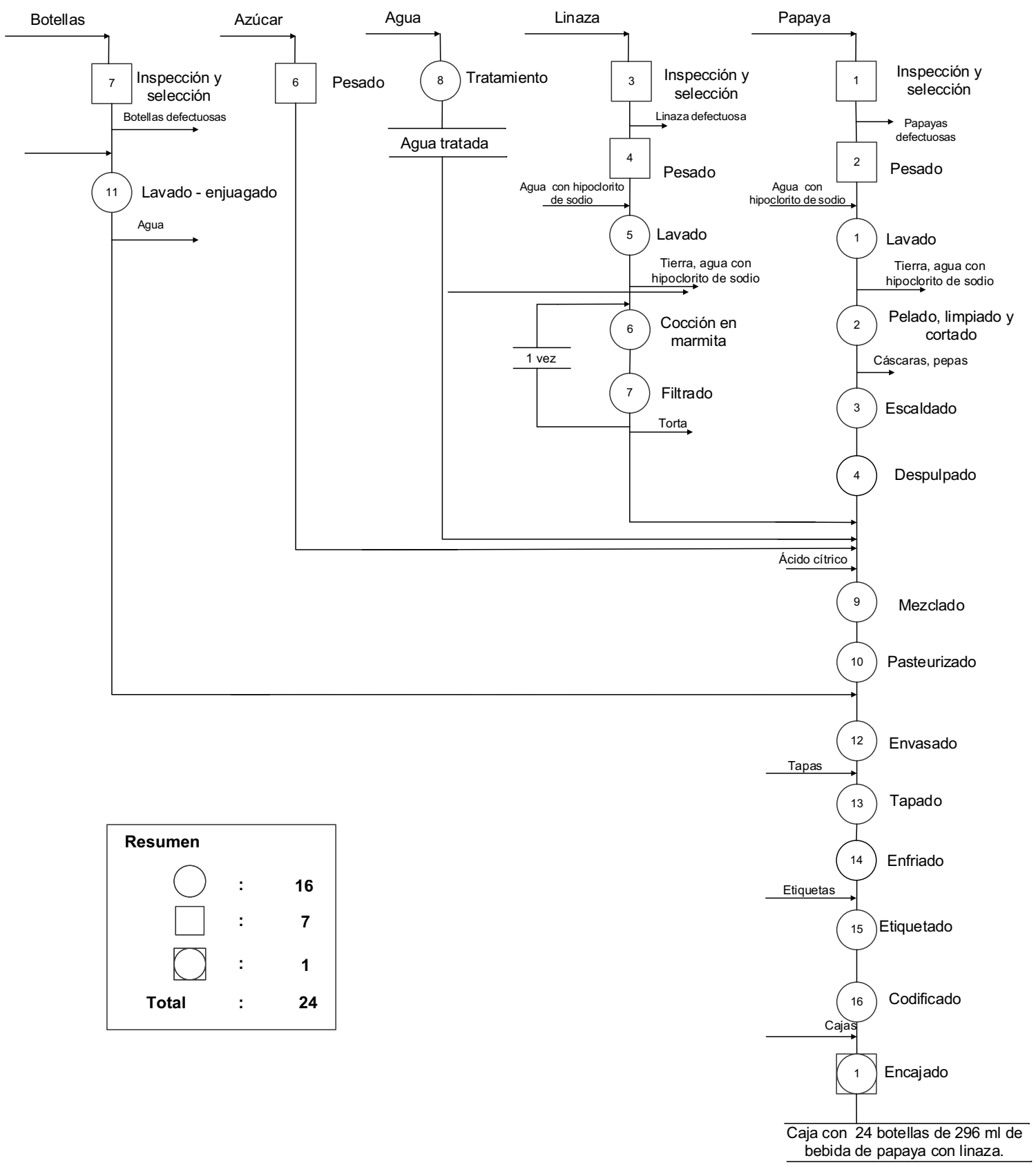

Figura 3. Diagrama de operaciones del proceso para la elaboración de bebida de papaya con linaza

\section{Elaboración propia}




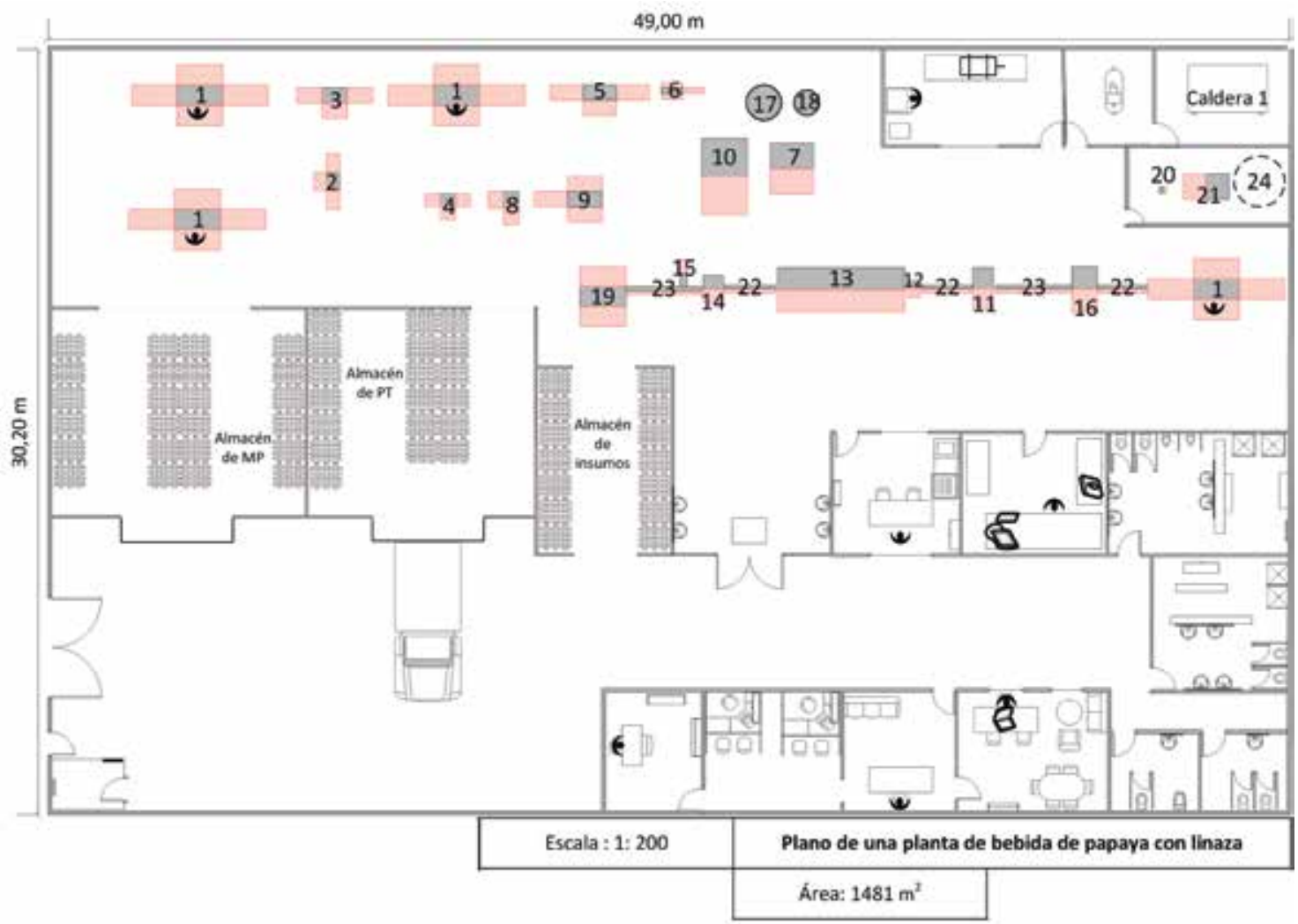

\begin{tabular}{|lll|}
\hline Leyenda & & \\
\hline 1. Mesa de selección y pelado & 9. Filtro vibratorio & 17. Tanque de almacenamiento 1 \\
2. Balanza industrial & 10. Tanque de mezclado & 18. Tanque de almacenamiento 2 \\
3. Lavadero grande & 11. Marmita & 19. Mesa de embalado \\
4. Lavadero pequeño & 12.Tapadora & 20. Filtro DUO \\
5. Equipo de escaldado & 13. Túnel de enfriamiento & 21. Esterilizador UV \\
6. Pulpeadora de frutas & 14. Etiquetadora manual & 22. Faja transportadora pequeña \\
7. Pasteurizadora & 15. Codificador & 23. Faja transportadora larga \\
8. Marmita & 16. Lavadora de botellas & 24. Tanque de agua \\
\hline
\end{tabular}

Figura 4. Plano de disposición de planta

Elaboración propia 


\subsection{Disposición de planta}

Para proponer la distribución de la planta de elaboración de bebida de papaya con linaza se calcula las áreas de cada zona, para lo que se utiliza el método de Guerchet, el cual sirve para determinar la superficie mínima requerida por las áreas de producción.

Una vez definida el área mínima, se analizan las actividades que se realizan en cada zona de trabajo, y se revisan los flujos de materiales y de productos terminados, para que estos tengan relación y no se genere un cruce con posible contaminación. Finalmente, se distribuyen las áreas con sus respectivas dimensiones, para construir el plano de distribución al detalle, como se muestra en la figura 4.

\section{ASPECTOS ECONÓMICOS Y FINANCIEROS}

\subsection{Inversiones}

Para el cálculo de la inversión total se tomó en cuenta los activos fijos tangibles e intangibles y el capital de trabajo, como se detalla en la tabla 11:

Tabla 11

Cálculo de la inversión total

\begin{tabular}{lr}
\hline Activos fijos tangibles & Monto (S/.) \\
\hline Terreno & 2488080 \\
Mueble, equipos y enseres & 23398 \\
Infraestructura del proyecto & 1224840 \\
Maquinaria y equipos & 276861,80 \\
Instalación de maquinaria & 42000 \\
Total activo fijo tangible & 055178,80 \\
Activos fijos intangibles & \\
Estudio de factibilidad & 22400 \\
Licencia de construcción & 500 \\
Marco Legal & 1800 \\
Gasto por capacitación del personal & 5300 \\
Total activos intangibles & 30000 \\
Total de activos fijos & $\mathbf{4 0 8 5} \mathbf{1 7 8 , 8 0}$ \\
Capital de trabajo & $\mathbf{1 1 8} \mathbf{4 9 9 , 6 1}$ \\
Inversión total & $\mathbf{4 2 0 3} \mathbf{6 7 8 , 4 1}$ \\
\hline
\end{tabular}

Elaboración propia 


\subsection{Financiamiento}

En este proyecto se considera que el accionista va a aportar el $50 \%$ de la inversión y el otro 50\% será financiado. Para el financiamiento se escogió el programa Probid. En la tabla 12 se presenta la forma como se calculó la tasa de interés.

Para calcular el costo promedio ponderado de capital (CPPC), primero se determina el valor del costo de oportunidad del accionista (COK), el cual se encuentra detallado en la tabla 13; siendo Rf (tasa libre de riesgos), $\mathrm{Rm}$ (rendimiento del mercado) y Beta (coeficiente de variabilidad).

\section{Tabla 12}

Tasa de interés del préstamo

\begin{tabular}{lc}
\hline Concepto & Tasa $\%$ \\
\hline VAC & 650 \\
Tasa & 7,13 \\
Comisiones & 1,75 \\
TEA & 15,38 \\
\hline
\end{tabular}

Fuente: Cofide (s. f.)

Elaboración propia
Tabla 13

Cálculo del COK

\begin{tabular}{lc}
\hline Rf & $5,00 \%$ \\
Beta & 0.91 \\
Rm & $18,00 \%$ \\
COK & $16,83 \%$ \\
\hline
\end{tabular}

Fuente: Gestión (s. f.), León (s. f.)

Elaboración propia

Una vez calculado el COK, se realiza el cálculo del CPPC, el cual se detalla en la tabla 14.

Tabla 14

Repartición de la inversión

\begin{tabular}{lcccc}
\hline & $\begin{array}{c}\text { Importe } \\
\text { S/. }\end{array}$ & $\begin{array}{c}\text { Participación } \\
\%\end{array}$ & $\begin{array}{c}\text { Tasa } \\
\%\end{array}$ & $\begin{array}{c}\text { CPPC } \\
\%\end{array}$ \\
\hline Capital propio & 2102683 & 50,0 & 16,83 & 8,42 \\
Financiamiento & 2102683 & 50,0 & 15,38 & 5,38 \\
\hline & & & CPPC & 13,80 \\
\hline
\end{tabular}

Elaboración propia 


\subsection{Análisis económico y financiero}

Para la evaluación económica se debe tener en cuenta que la inversión necesaria será aportada por los accionistas al $100 \%$, para este análisis se utiliza el COK hallado en la tabla 13; obteniéndose los siguientes resultados:

- VANE: S/.411 679,98

- TIRE: $18,82 \%$

- B/C: S/.1098

- PR: 9,58 años

Asimismo, para la evaluación financiera se va a utilizar un financiamiento externo y el costo promedio ponderado (CPPC) hallado en la tabla 14, obteniéndose los siguientes resultados:

- VANF: S/.1 440 309,20

- TIRF: $24,53 \%$

- B/C: S/.1,69

- PR: 7,49 años

\section{IMPACTO SOCIAL DEL PROYECTO}

Según las proyecciones brindadas por el INEI (s. f.b), Ate tiene una población de 592345 habitantes, de los cuales 210446 pertenecen a la PEA. Cabe resaltar que de todos estos habitantes el 96,8 \% se encuentra dentro de la PEA ocupada, de los cuales solo el 17,6 \% labora en empresas manufactureras. Debido a esto, se considera que uno de los impactos positivos del proyecto es la generación de trabajo al contratar los servicios de estas personas.

Asimismo, se presenta el cálculo de algunos indicadores con el fin de poder cuantificar el crecimiento económico desde la perspectiva social, teniendo en cuenta que el valor agregado es de S/.11 939 666, el cual mide el impacto social que genera el proyecto a partir de su operación.

De acuerdo al resultado obtenido en la tabla 15, se señala que el proyecto es socialmente viable, debido a que la relación valor agregadoinversión es mayor a 1 , con esto se puede concluir que por cada sol invertido en el proyecto se genera un valor agregado de $\mathrm{S} / .2,84$. 
Tabla 15

Relación producto capital

\begin{tabular}{lc}
\hline & Valor \\
\hline Valor agregado & S/.11939666 \\
Inversión total & S/.4 203678,41 \\
P/C & 2,84 veces \\
\hline
\end{tabular}

Elaboración propia

Tabla 16

Densidad de capital

\begin{tabular}{lr}
\hline & \multicolumn{1}{c}{ Valor } \\
\hline Inversión total & $\mathrm{S} / .4203678,41$ \\
Número de trabajadores & 40 \\
Inversión / \# Trab. & $\mathrm{S} / .105$ 091,96/Trab.
\end{tabular}

Elaboración propia

De igual manera, se determinó que para generar un puesto de trabajo se debe invertir los S/.105 091,96.

Finalmente, la relación inversión total-valor agregado indica que para generar un sol de valor agregado se requiere S/.0,35.

Tabla 17

Intensidad de capital

\begin{tabular}{lr}
\hline & \multicolumn{1}{c}{ Valor } \\
\hline Valor agregado & $\mathrm{S} / .11939666$ \\
Inversión total & $\mathrm{S} / .4203678,41$ \\
I.C. & 0,35 veces \\
\hline
\end{tabular}

Elaboración propia

\section{CONCLUSIONES}

- La ejecución del proyecto de implementación de una planta para la elaboración de bebida de papaya con linaza es factible, ya que en el mercado nacional existe una creciente demanda hacia el consumo de los jugos, néctares y bebidas. Además, es viable tecnológica, económica, financiera, medioambiental y socialmente. 
- El estudio está enfocado en los sectores A, B y C del mercado peruano que tengan más de 13 años, ya que estos sectores presentan un mayor gasto en alimentación. No obstante, el proyecto solo se comercializará en Lima metropolitana, por tener la mayor población de los sectores mencionados. De acuerdo con la segmentación realizada, se obtuvo una demanda de 1794083 botellas de $296 \mathrm{ml}$; y se estableció un precio de $\mathrm{S} / .2,30$.

- En el tamaño de planta, el limitante es la tecnología, la cual es determinada por la capacidad de producción de la operación cuello de botella del proceso, y corresponde a la operación de filtrado. Esta operación determina la capacidad instalada de la planta, cuyo valor es de 762636,81 l/año.

- La inversión requerida para el proyecto es de S/.4 203 678,41; su financiación será de la siguiente manera: $50 \%$ por los accionistas y el otro $50 \%$ por Cofide, con un préstamo de cuotas crecientes, con un año de gracia parcial por los próximos 10 años de operación.

- En lo referente a la evaluación financiera del proyecto, se obtuvo un VANF de S/. 1440 309,20, así como una TIRF del 24,53 \%, lo cual respalda la viabilidad del proyecto. Asimismo, se realizó un análisis de sensibilidad en el cual se considera como variables el precio, el costo de la papaya, el costo de la linaza y la tasa del préstamo.

- En la evaluación social del proyecto resultó un impacto positivo, ya que aumenta las oportunidades laborales. Esto se corrobora con el cálculo del índice de valor agregado de S/.11939666.

\section{RECOMENDACIONES}

- Para ajustar las proporciones de los ingredientes de la bebida se deben realizar focus group, en los cuales se den a probar a cada uno de los participantes la formulación obtenida en el estudio de prefactibilidad para ver si ellos aceptan el sabor, en caso de que la respuesta sea negativa se deben realizar nuevas pruebas para mejorar la formulación.

- Se sugiere realizar siempre las pruebas del laboratorio del producto por elaborar, con el objetivo de asegurarse que este realmente se puede producir y así anotar información relevante para el proyecto.

- Se deberá evaluar técnica, económica y financieramente, la posibilidad de incursionar en el mercado, con un producto similar pero 
con diferente sabor, como bebida de maracuyá con linaza, pera con linaza, entre otros.

- Se debe analizar la posibilidad de tercerizar los diversos servicios para concentrar el esfuerzo de la organización en aquellas actividades que realmente le agreguen valor.

\section{REFERENCIAS}

Aravind. G., Debjit Bhowmik, Duraivel, S., y Harish, G. (2013). Traditional and medicinal uses of Carica papaya. IDP. Journal of Medecinal Plants Studies, 1, 7-14. Recuperado de http:// plantsjournal.com/vol1Issue1/Issue_jan_2013/2.pdf.

BCRP - Banco Central de Reserva del Perú. (s. f.). memoria 2009. Recuperado de http://www.bcrp.gob.pe/publicaciones/memoriaanual/memoria-2009.html

Castro Ríos, K. (2011). Tecnología de alimentos. Bogotá: Ediciones de la U.

Cofide - Corporación Financiera de Desarrollo. (s. f.). Tasa de intereses y comisiones a las instituciones financieras intermediarias de los programas y líneas de financiamiento Cofide. Recuperado de www.cofide.com.pe

Dávila, R. (2009). Gel antirreumático y alimentos a partir de residuos de papaya. Journalmex. Recuperado de http://journalmex.wordpress. com/2009/02/02/gel-antirreumatico-y-alimentos-a-partir-deresiduos-de-papaya/

Driessen, S. (s. f). Vacuum sealing food at home safely (University of Minnesota). Recuperado de http://www1.extension.umn.edu/food/ food-safety/preserving/vacuum-sealing/

FAO - Food and Agricultura Organization of the United Nations. (s. f.). Elaboración de néctar de frutas tropicales. Recuperado de www. fao.org/

Gestión. (s. f.). Locales industriales en Ate tienen los precios más altos en Lima. Diario Gestión. Lima. Recuperado de www.gestion.pe/ inmobiliaria/locales-industriales-ate-tienen-precios-mas-altoslima-2057734.

Indecopi - Instituto Nacional de Defensa de la Competencia y de la Protección de la Propiedad Intelectual. (2008). Norma de Metrológica Peruana: cantidad de producto en preenvases. Lima: Indecopi. 
Indecopi - Instituto Nacional de Defensa de la Competencia y de la Protección de la Propiedad Intelectual. (2009). Norma técnica peruana: azúcar, azúcar refinado. Requisitos. Lima: Indecopi.

INEI - Instituto Nacional de Estadística e Informática (s. f.a). Perú en números (proyección del crecimiento del PBI). Recuperado de http://www.inei.gob.pe/estadisticas/indice-tematico/economia/

INEI - Instituto Nacional de Estadística e Informática (s. f.b). Estimaciones y proyecciones de la población por grupos quinquenales de edad, según departamentos, provincias y distritos 2005-2015. Recuperado de http://www.inei.gob.pe/biblioineipub/bancopub/ Est/Lib0842/index.htm.

Ipsos-Apoyo Opinión y Mercado. (2012a). Informe gerencial de marketing: Estadística poblacional. Marketing Data Plus. Recuperado de http://www.ipsos_apoyo.org.pe

Ipsos-Apoyo Opinión y Mercado. (2012b). Informe gerencial de marketing: Perfil ama de casa 2010. Marketing Data Plus. Recuperado de http://www.ipsos_apoyo.org.pe

Ipsos-Apoyo Opinión y Mercado. (2012c). Informe gerencial de marketing: Tendencia en salud y alimentación 2009. Marketing Data Plus. Recuperado de http://www.ipsos_apoyo.org.pe

Ipsos-Apoyo Opinión y Mercado. (2012d). Informe gerencial de marketing: Niveles socioeconómicos 2012. Marketing Data Plus. Recuperado de http://www.ipsos_apoyo.org.pe

Ipsos-Apoyo Opinión y Mercado. (2012e). Informe gerencial de marketing: Liderazgo en productos comestibles 2012. Marketing Data Plus. Recuperado de http://www.ipsos-apoyo.org.pe

León, C. (s. f.). Costo de capital en los países emergentes: análisis para la industria de alimentos peruana. Chiclayo: Universidad Católica de Santo Toribio de Mogrovejo. Recuperado de http://issuu.com/ flumen.usat/docs/dt024-ckalimentos

Maximixe Consult S. A. (2012). Informe de jugos de fruta 2012. Caser: Riesgos de mercado. Lima.

Ministerio de la Producción. (2012). Anuario estadístico 2012. Recuperado de http://www.produce.gob.pe/portal/ 
Pardo, J., y Urquizo, K. (2014). Estudio de prefactibilidad para la implementación de una planta de elaboración de bebida de papaya (Carica papaya) con linaza (Linum usitatissimum). (Tesis para optar el título de ingeniero industrial). Universidad de Lima. Perú.

Sunat. (1997-2012). Clasificación arancelaria. Recuperado de http:// www.sunat.gob.pe/

United Nations Statistics Division. (2012). Clasificaciones disponibles -CIIU. Recuperado de http://unstats.un.org/unsd/cr/registry/ regcs.asp? $\mathrm{Cl}=17 \& \mathrm{Co}=1554 \& \mathrm{Lg}=3 \& \mathrm{prn}=$ yes

Van Saafelo, K. (2012). Recomendaciones nutricionales para disminuir los triglicéridos. Geosalud. Recuperado de http://www.geosalud. com/nutrición/triglicéridos/htm 
